\title{
PERFIL DE SAÚDE DOS IDOSOS ATENDIDOS NAS UNIDADES BÁSICAS DE SAÚDE DA FAMÍLIA (UBSF) EM ITUIUTABA, MINAS GERAIS
}

\author{
HEALTH PROFILE OF THE ELDERLY ATTENDED AT FAMILY HEALTH BASIC UNITS (UBSF) IN \\ ITUIUTABA, MINAS GERAIS
}

\author{
Janyne Vilarinho Melo ${ }^{\mathrm{a}^{*}}$, Ana Lúcia de Medeiros Santos ${ }^{\mathrm{b}^{*}}$, Lina Rodrigues de Faria ${ }^{\mathrm{c}^{* *}}$, \\ Waneska Alexandra Alves ${ }^{\mathrm{d}^{* * * *}}$, Alexandre Azenha Alves de Rezende $\mathrm{e}^{*}$, Luciana Karen Calábria ${ }^{\mathrm{f}^{*}}$ \\ ajanyne_mello@hotmail.com, bannamedeiros94@hotmail.com, clinafaria1964@gmail.com, dwaneska.alves@ufjf.edu.br, \\ eazenha@ufu.br, ${ }^{\circ} \mathrm{Ikcalabria@ufu.br,}$ \\ *Universidade Federal de Uberlândia - Ituiutaba (MG), Brasil \\ **Universidade Federal do Sul da Bahia - Porto Seguro (BA), Brasil \\ ***Universidade Federal de Juiz de Fora - Governador Valadares (MG), Brasil
}

Data de recebimento do artigo: 01/06/2017

Data de aceite do artigo: 01/08/2017

\section{RESUMO}

Introdução: $\mathrm{O}$ processo de envelhecimento se caracteriza por alteraçôes biológicas que ocorrem ao longo da vida. Essas alteraçôes podem provocar doenças ou disfunçôes orgânicas, entre as quais se destacam as doenças crônicas não transmissíveis (DCNT). Objetivo: Considerando a importância das DCNT, o estudo caracterizou os perfis socioeconômico e demográfico dos idosos atendidos em Unidades Básicas de Saúde da Família (UBSF) no município de Ituiutaba/MG. Material e métodos: Realizou-se um estudo transversal descritivo, em que 149 idosos atendidos em onze UBSF foram entrevistados através de questionário semiestruturado. Resultados: A idade média dos participantes foi de setenta anos, sendo a maioria do gênero feminino, alfabetizada e com até quatro anos de estudo. Aproximadamente $90 \%$ dos idosos declararam uso de medicamentos alopáticos, $47 \%$ faziam uso de plantas medicinais e $19,5 \%$ de fitoterápicos. Somente $14,7 \%$ dos idosos afirmaram o uso concomitante dessas três modalidades de tratamento. A maioria dos entrevistados utilizava de dois a quatro medicamentos alopáticos $(51,7 \%)$. Em relação às doenças autorreferidas, as mais prevalentes foram as do sistema circulatório $(75,8 \%)$, destacando-se a hipertensão arterial $(72,5 \%)$, além de doenças no sistema osteomuscular $(41,6 \%)$ e diabetes mellitus $(39,9 \%)$. Conclusáo: De acordo com os dados obtidos, foi possível realizar um diagnóstico dos idosos atendidos nas UBSF de Ituiutaba/MG. Esses dados são essenciais para implementação de políticas públicas, sociais e educacionais voltadas a essa população, a fim de atender às necessidades socioeconômicas e comportamentais, visando à promoçáo de um envelhecimento saudável e ativo.

Palavras-chave: Envelhecimento; doenças crônicas; uso de medicamentos; epidemiologia.

\section{ABSTRACT}

Introduction: The aging process is characterized by biological changes that occur throughout life. These changes can cause diseases or organic dysfunctions, in which Chronic Non-communicable Diseases (DCNT) stand out. Objective: Considering the importance of DCNT, the study identified the socioeconomic and demographic profiles of the elderly assisted at Family Health Basic Units (UBSF) in the municipality of Ituiutaba/MG. Material and methods: A descriptive cross-sectional study was carried out on 149 elderly people assisted in 11 UBSF, who were interviewed through a semi-structured questionnaire. Results: Their mean age was 70 years old, most of them were of the female gender, literate and with up to four years of study. Approximately $90 \%$ of the elderly reported the use of allopathic drugs, $47 \%$ used herbal medicines and $19.5 \%$ used herbal medicines. Only $14.7 \%$ of the elderlies reported the concomitant use of these three treatment modalities. Most of the interviewees used 2 to 4 allopathic medications (51.7\%). Regarding the self-reported diseases, the most prevalent diseases were those from the circulatory system (75.8\%), especially hypertension (72.5\%), as well as 
musculoskeletal system diseases (41.6\%) and diabetes mellitus (39.9\%). Conclusion: According to the data obtained, it was possible to perform a diagnosis of the elderly assisted at the UBSF of Ituiutaba/MG. These data are essential for the implementation of public, social and educational policies directed to this population, in order to meet the socio-economic and behavioral needs, aiming to promote a healthy and active aging.

Keywords: Aging; chronic diseases; drug utilization; epidemiology.

\section{Introdução}

A preocupação com as condiçóes de saúde do idoso tem motivado o desenvolvimento de vários estudos sobre o envelhecimento humano, em especial, sobre políticas públicas na esfera do chamado envelhecimento ativo e saudável, políticas sociais voltadas para o bem-estar dos idosos e para a qualidade dos serviços prestados a eles, além de outros temas, como relação entre pobreza, ambiente e saúde, redes sociais de apoio à pessoa idosa, conflitos intergeracionais e apoio familiar ${ }^{1-5}$.

Longe de representar uma sociedade envelhecida, o Brasil experimenta, não obstante, um processo de transição demográfica que altera seu perfil epidemiológico, com o envelhecimento da população, resultado do aumento da expectativa de vida e da reduçáo da taxa de natalidade ${ }^{6,7}$. O Ministério do Planejamento, Orçamento e Gestão ${ }^{8}$ evidenciou que, entre 2001 e 2011, o número de idosos de sessenta anos ou mais passou de $15,5 \mathrm{mi}-$ lhóes para 23,5 milhóes. Além disso, com o aumento da expectativa de vida, estima-se que essa população passará de $13,8 \%$ até 2020 e de $33,8 \%$ até $2060^{\circ}$.

O envelhecimento demográfico, junto com a transição epidemiológica, aumenta a prevalência das doenças crônicas não transmissíveis (DCNT), uma das principais causas de morbidade e mortalidade entre idosos no país. As condições crônicas constituem problemas de saúde que demandam cuidados e gerenciamento permanentes por longo período ${ }^{7}$.

É notório que acompanha o envelhecimento uma maior demanda por serviços de saúde e o aumento no uso de medicamentos, o que predispóe essa população aos riscos da prática de polifarmácia e, consequentemente, aos efeitos adversos dos medicamentos, além de maiores taxas de internações hospitalares quando comparadas a outras faixas etárias ${ }^{10-14}$. Assim, os serviços de saúde desempenham papel fundamental na prevenção e na diminuição das doenças crônicas e de suas complicaçôes. A elevada prevalência das condiçóes crônicas tem exigido transformaçóes na organização dos serviços de saúde, de modo a promover estratégias de combate às doenças e prevenção de sequelas ${ }^{13}$.

As DCNT são definidas como morbidades irreversíveis de longo curso clínico e estão relacionadas à fragilidade natural da pessoa idosa. Alguns fatores sociais, econômicos, ambientais e culturais contribuem para seu desenvolvimento, a saber: desigualdade social, diferenças no acesso aos serviços e às informaçôes de saúde (devido a condiçóes geográficas), baixa escolaridade e fatores de risco ambiental modificáveis ${ }^{6}$.

Um levantamento promovido pelo Instituto Brasileiro de Geografia e Estatística (IBGE) em 2013 alertou que as DCNT são responsáveis por $72 \%$ das causas de morte na população idosa, com destaque para doenças do aparelho circulatório $(31,3 \%)$, câncer $(16,3 \%)$, diabetes $(5,2 \%)$ e doença respiratória crônica $(5,8 \%)^{15}$. Além disso, os principais fatores de risco estão associados ao uso de tabaco, à inatividade física e ao consumo abusivo de álcool, bem como à alimentação não saudável, incluindo a ingestão de alimentos com gorduras saturadas e ácidos graxos e o consumo elevado de sal, que contribuem para quadros de hipertensão e doenças cardíacas ${ }^{15}$. O consumo frequente e excessivo de doces e açúcares pode aumentar a prevalência de sobrepeso, acarretando obesidade e intolerância à glicose, o que provoca diabetes mellitus tipo $2^{16}$.

Algumas políticas públicas de saúde no Brasil têm enfatizado estratégias para combater as DCNT de modo preventivo, com açóes de diversos setores ${ }^{17}$. No município de Ituiutaba/MG, a atenção está voltada para a saúde da população idosa por meio das Unidades Básicas de Saúde da Família (UBSF), que possuem um planejamento estratégico para facilitar o acesso da população ao serviço de saúde com o apoio dos Programas de Saúde da Família (PSF) implantados nas áreas periféricas, ajudando, assim, no fluxo de atendimento das $\mathrm{UBSF}^{18}$. Assim, este estudo analisou a atual situação da populaçáo idosa residente no município de Ituiutaba/ MG e atendida nas UBSF, traçando o perfil de saúde dessa populaçáo e investigando o uso de medicamentos alopáticos, plantas medicinais e fitoterápicos.

\section{Metodologia}

Um estudo transversal e descritivo foi realizado com 149 idosos não institucionalizados, atendidos pelas equipes de Saúde da Família do município de Ituiutaba/MG em 2015. A Saúde da Família está presente em onze bairros periféricos do município de Ituiutaba, sendo eles: Alvorada, Camargo, Independência, Jardim do Rosário, Pirapitinga, Natal, Novo Horizonte, Novo Tempo 2, 
Santa Maria, Setor Norte e Sol Nascente (Figura 1). A pesquisa foi aprovada pelo Comitê de Ética em Pesquisa Humana, sob o parecer no 329.774 (CEP/UFJF) e faz parte do Programa de Atençáo Preventiva e Educativa em Saúde do Idoso do Núcleo de Estudos da Pessoa Idosa, em parceria com a Universidade Federal de Juiz de Fora, campus Governador Valadares. Os participantes que concordaram em colaborar como voluntários, por livre e espontânea vontade, assinaram o Termo de Consentimento Livre e Esclarecido (TCLE) contendo informaçóes referentes ao desenvolvimento da pesquisa. Os critérios de inclusão foram: ser atendido em uma das onze UBSF, ter idade igual ou superior a sessenta anos, ter interesse em participar e assinar o termo. Os critérios de exclusão foram: ter idade inferior a sessenta anos, não ter interesse em participar da pesquisa e não assinar o TCLE.

Figura 1: Mapa da cidade de Ituiutaba/MG destacando a localização das Unidades Básicas de Saúde da Família (UBSF), 2015

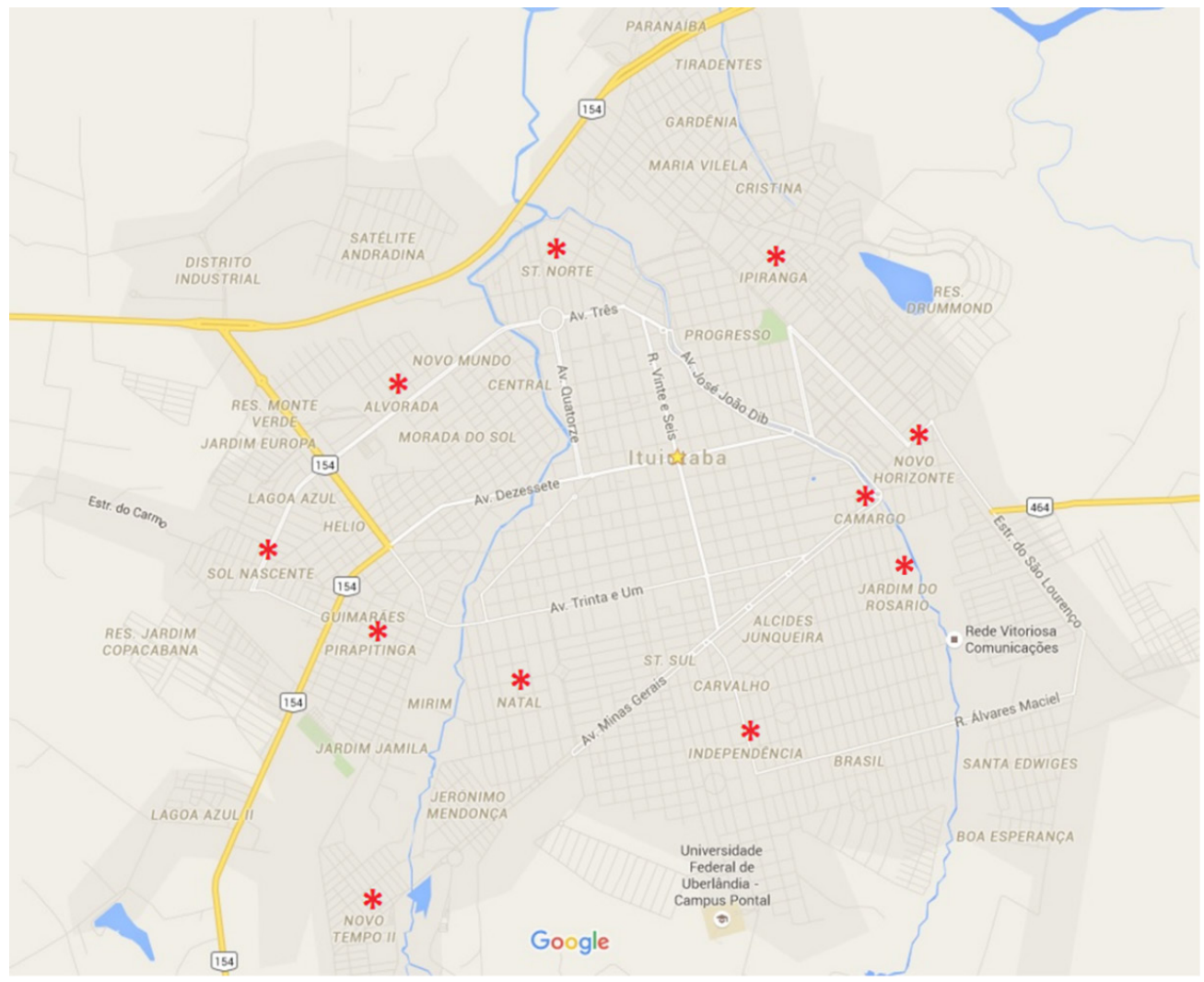

Fonte: GoogleMaps (2015).

A coleta de dados foi realizada utilizando um questionário semiestruturado com variáveis sociodemográficas (idade, gênero, e escolaridade), perguntas referentes à saúde (diagnóstico médico de doença crônica, doenças autorreferidas no aparelho circulatório, no sistema osteomuscular, no aparelho respiratório, transtornos mentais ou comportamentais, doenças endócrinas e outras; o tipo de diabetes mellitus; uso contínuo, nome e quantidade de medicamentos; informação sobre queda ou lesão de pele; uso de próteses dentária e ortopédica; utilização de planta medicinal e fitoterapia) e a hábitos de vida (autopercepção da saúde como boa, ótima, razoável ou ruim).

Os dados foram tabulados utilizando o programa computacional Microsoft Office Excel 2007. Cada variável foi avaliada por meio de estatística descritiva, analisando o número amostral; frequências absoluta (n) e relativa (\%); média e desvio padrão. A correlaçáo de Pearson foi realizada por meio do programa BioEstat, versão 5.0 , com nível de confiança de $95 \%$, considerando $p<0,05$.

\section{Resultados e discussão}

A idade dos participantes variou de 60 a 85 anos com média de 70,0 $\pm 6,1$ anos. As mulheres tiveram maior frequência na amostra total $(66,4 \%)$ em relação aos homens $(35,6 \%)$. A maioria dos idosos entrevistados tem até quatro 
anos de estudos (52,3\%). Para Santos et al. ${ }^{19}$, a escolaridade é um fator considerável para a qualidade de vida, visto que pessoas com grau de escolaridade mais avançado assimilam melhor as informaçóes do dia a dia, o que proporciona melhores condiçóes de vida social e econômica, além de maior facilidade de acesso a serviços de saúde.

Tabela 1: Dados autorreferidos de registros de quedas, fraturas ósseas e uso de prótese ortopédica de idosos atendidos nas Unidades Básicas de Saúde da Família (UBSF), Ituiutaba/MG, 2015.

\begin{tabular}{|c|c|c|c|c|c|c|c|c|c|c|c|c|c|c|c|}
\hline \multirow[t]{2}{*}{ Variáveis } & \multicolumn{2}{|c|}{$\begin{array}{c}\text { Total } \\
(n=149)\end{array}$} & $\begin{array}{l}\text { UBSF } 1 \\
(n=14)\end{array}$ & $\begin{array}{l}\text { UBSF } 2 \\
(n=13)\end{array}$ & $\begin{array}{l}\text { UBSF } 3 \\
(n=13)\end{array}$ & $\begin{array}{l}\text { UBSF } 4 \\
(n=13)\end{array}$ & $\begin{array}{l}\text { UBSF } 5 \\
(\mathrm{n}=15)\end{array}$ & $\begin{array}{l}\text { UBSF } 6 \\
(n=13)\end{array}$ & $\begin{array}{l}\text { UBSF } 7 \\
(\mathbf{n}=14)\end{array}$ & $\begin{array}{l}\text { UBSF } 8 \\
(\mathrm{n}=14)\end{array}$ & $\begin{array}{l}\text { UBSF } 9 \\
(n=13)\end{array}$ & \multicolumn{2}{|c|}{$\begin{array}{l}\text { UBSF } 10 \\
(\mathrm{n}=14)\end{array}$} & \multicolumn{2}{|c|}{$\begin{array}{c}\text { UBSF } 11 \\
(n=13)\end{array}$} \\
\hline & $n$ & 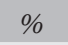 & $n$ & $\%$ & $\%$ & 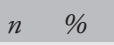 & $n$ & $n \quad \%$ & 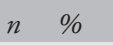 & (2) & 0 & 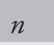 & & - & $\%$ \\
\hline & & & 6 & 0 & 5 & t & 853 & 5 & 35,7 & 42,9 & 15,4 & & & 4 & \\
\hline & 6 & & 1 & 0,0 & - & 0 , & 2 & 3 & 14,3 & 71 & & & & & \\
\hline topearca & 1 & 7 & 0,0 & 0,0 & 0,0 & 0,0 & $1 \quad 6,7$ & 0,0 & 7,1 & 0,0 & 7,7 & & 0,0 & & 5,4 \\
\hline
\end{tabular}

Fonte: Elaborado pelos autores (2015).

De acordo com os dados da Tabela 1, 32,9\% dos entrevistados relataram quedas e $9,4 \%$ fraturas, com destaque para as UBSF 3, 5, e 6, que apresentaram frequências acima da média. Os idosos que mencionaram ter sofrido alguma fratura citaram pernas, braços, costelas, dedos, pés, coluna e cabeça como locais atingidos. Segundo Kuznier et al. ${ }^{20}$, quedas são capazes de gerar aumento no nível de dependência do idoso e ocasionar uma preocupação específica, devido à possibilidade de afetar a capacidade funcional. O estudo de Siqueira et al. ${ }^{21}$, realizado com 4.003 idosos atendidos em Unidades Básicas de Saúde de sete estados brasileiros (Rio Grande do Sul, Santa Catarina, Alagoas, Pernambuco, Paraíba, Rio Grande do Norte e Piauí), revela a prevalência de
$34,8 \%$ de quedas entre os idosos, das quais $12,8 \%$ tiveram como consequência fraturas de braços, pulso, pés, costelas, mão, fêmur e/ou nariz. Esses resultados contrariam os encontrados por $\mathrm{Maciel}^{22}$, em que as quedas foram registradas para um terço dos idosos brasileiros que vivem em comunidade.

Alguns estudos confirmam que a fratura mais comum em idosos é a de fêmur ${ }^{23,24}$. O uso de prótese ortopédica foi restrito a $2,7 \%$ da população entrevistada (Tabela 1) e o fêmur foi o único local atingido. Identificou-se também baixa frequência do uso de próteses em idosos do Núcleo de Apoio à Saúde da Família de Montes Claros/ MG, onde somente 3\% dos 99 idosos que participaram da pesquisa relataram uso de prótese ortopédica ${ }^{25}$.

Tabela 2: Perfil do uso de medicamentos pelos idosos atendidos nas Unidades Básicas de Saúde da Família (UBSF), Ituiutaba/MG, 2015

\begin{tabular}{|c|c|c|c|c|c|c|c|c|c|c|c|c|c|c|c|c|c|c|c|c|c|c|c|c|}
\hline \multirow[t]{2}{*}{ Variáveis } & \multicolumn{2}{|c|}{$\begin{array}{c}\text { Total } \\
(n=149)\end{array}$} & \multicolumn{2}{|c|}{$\begin{array}{l}\text { UBSF } 1 \\
(n=14)\end{array}$} & \multicolumn{2}{|c|}{$\begin{array}{l}\text { UBSF } 2 \\
(\mathbf{n}=13)\end{array}$} & \multicolumn{2}{|c|}{$\begin{array}{l}\text { UBSF } 3 \\
(n=13)\end{array}$} & \multicolumn{2}{|c|}{$\begin{array}{l}\text { UBSF } 4 \\
(n=13)\end{array}$} & \multicolumn{2}{|c|}{$\begin{array}{l}\text { UBSF } 5 \\
(\mathrm{n}=15)\end{array}$} & \multicolumn{2}{|c|}{$\begin{array}{l}\text { UBSF } 6 \\
(n=13)\end{array}$} & \multicolumn{2}{|c|}{$\begin{array}{l}\text { UBSF } 7 \\
(\mathbf{n}=14)\end{array}$} & \multicolumn{2}{|c|}{$\begin{array}{l}\text { UBSF } 8 \\
(n=14)\end{array}$} & \multicolumn{2}{|c|}{$\begin{array}{l}\text { UBSF } 9 \\
(n=13)\end{array}$} & \multicolumn{2}{|c|}{$\begin{array}{c}\text { UBSF } 10 \\
(n=14)\end{array}$} & \multicolumn{2}{|c|}{$\begin{array}{l}\text { UBSF } 11 \\
(\mathrm{n}=13)\end{array}$} \\
\hline & $n$ & $\%$ & $n$ & $\%$ & $n$ & $\%$ & $n$ & $\%$ & $n$ & $\%$ & $n$ & $n$ & $\%$ & $\%$ & $n$ & $\%$ & $n$ & $\%$ & $n$ & $\%$ & $n$ & $\%$ & $n$ & $\%$ \\
\hline \multicolumn{25}{|l|}{ Alopáticos } \\
\hline 1 & 19 & 8 & 3 & 1,4 & 2 & 15,4 & 2 & 15,4 & 6 & 42,8 & 2 & 13,3 & 1 & 7,6 & 1 & 7,2 & 4 & 42,9 & 4 & 0,8 & 4 & & 1 & 7,6 \\
\hline $\begin{array}{l}2 \text { a } 4 \\
\text { (Pré-polifarmácia) }\end{array}$ & 77 & 51,7 & 7 & 50,0 & 10 & 76,9 & 4 & 28,6 & 6 & 42,8 & 8 & 53,3 & 6 & 46,2 & 9 & 64,3 & 7 & 50,0 & 7 & 53,8 & 6 & 42,8 & 7 & 53,8 \\
\hline $\begin{array}{l}5 \text { ou mais } \\
\text { (Polifarmácia) }\end{array}$ & 39 & 26,2 & 4 & 28,6 & 1 & 7,6 & 7 & 53,8 & 1 & 7,7 & 5 & 33,3 & 6 & 46,2 & 4 & 28,6 & 3 & 7 & 2 & 15,4 & 4 & 28,6 & 2 & 15,4 \\
\hline \multicolumn{25}{|l|}{ Fitoterápicos } \\
\hline Usam & 29 & 19,5 & 3 & 21,4 & 1 & 7,6 & 0 & 0 & 0 & 0 & 3 & 20 & 1 & 37.6 & 7 & 50 & 6 & 42,8 & 3 & 23,1 & J & 35,7 & 3 & 23,1 \\
\hline Não usam & 120 & 80,5 & 11 & 78,6 & 12 & 92,3 & 13 & 100 & 13 & 100 & 11 & 73,3 & 12 & 92,4 & 7 & 50 & 8 & 57,2 & 10 & 76,9 & O & 3 & 10 & 76,9 \\
\hline \multicolumn{25}{|l|}{ Plantas medicinais } \\
\hline Usam & 70 & 47,0 & 8 & 57,2 & 4 & 30,8 & 8 & 61,5 & 7 & 53,8 & 8 & 53,3 & 5 & 38,5 & 3 & 21,4 & 10 & 71,4 & 7 & 53,8 & (1) & ,3 & 4 & 30,8 \\
\hline Não usam & 79 & 53,0 & 6 & 42,8 & 9 & 69,2 & 5 & 38,5 & 6 & 46,2 & 7 & 46.6 & 8 & 61,5 & 11 & 78,6 & 4 & 28,6 & 6 & 46,2 & 5 & 35,7 & 9 & 69,2 \\
\hline $\begin{array}{l}3 \text { ou mais } \\
\text { (Poliherbácia) }\end{array}$ & 40 & 26,8 & 5 & 35,7 & 1 & 7,7 & 6 & 46,2 & 6 & 46,2 & 5 & 33,3 & 3 & 23,1 & 2 & 14,3 & 3 & 21,4 & 3 & 23,1 & 5 & 35,7 & 1 & 7,7 \\
\hline \multicolumn{25}{|c|}{ Tratamentos concomitantes } \\
\hline $\mathrm{AL}+\mathrm{PM}$ & 64 & 42,9 & 8 & 57,2 & 3 & 23,1 & 7 & 53,8 & 5 & 38,5 & 8 & 53,3 & 5 & 38,5 & 3 & 21,4 & 7 & 50 & 6 & 46,2 & 0 & 57,2 & 4 & 30,8 \\
\hline & 29 & 19,5 & 3 & 21,4 & 1 & 7,7 & 0 & 0 & 0 & 0,0 & 3 & 20 & 1 & 7,6 & 7 & 50 & 6 & 42,9 & 3 & 23,1 & 2 & 14,3 & 3 & 23,1 \\
\hline $\mathrm{AL}+\mathrm{PM}+\mathrm{FIT}$ & 22 & 14,7 & 3 & 21,4 & 1 & 7,7 & 0 & 0 & 0 & 0,0 & 3 & 20 & 1 & 7,6 & 3 & 21,4 & 4 & 28,6 & 3 & 23,1 & 2 & 14,3 & 3 & 23,1 \\
\hline
\end{tabular}

AL: medicamentos alopáticos; PM: plantas medicinais; FIT: fitoterápicos.

Fonte: Elaborado pelos autores (2015). 
A Tabela 2 evidencia o uso de medicamentos, plantas medicinais e fitoterápicos pelos idosos entrevistados. Em torno de 90,6\% declararam utilizar medicamentos alopáticos, $47 \%$ fazem uso de plantas medicinais e $19,5 \%$ de fitoterápicos. Desses, $42,9 \%$ afirmaram combinar o uso de alopáticos e plantas medicinais, e 19,5\% utilizam concomitantemente alopáticos e fitoterápicos. Somente $14,7 \%$ dos idosos afirmaram combinar as três modalidades de tratamento. Essa alta frequência do uso de medicamentos alopáticos também é observada em outros estudos ${ }^{26,27}$. Em contrapartida, o uso de plantas medicinais e fitoterápicos foi registrado por Machado et al. ${ }^{28}$, respectivamente, em $76,7 \%$ e $5,5 \%$ dos idosos que frequentam o grupo de Atividade Física e Recreativa para a Terceira Idade (Afrid) da Universidade Federal de Uberlândia, Minas Gerais, (UFU/MG).

Por meio de análise estatística, foi possível constatar que quanto maior o número de plantas medicinais e de medicamentos alopáticos utilizados pelos idosos entrevistados nas UBSF em Ituiutaba/MG, pior é sua percepção da própria saúde $(p \leq 0,0001)$. O uso de fitoterápicos e plantas medicinais foi maior entre as mulheres, das quais $72,5 \%$ utilizam plantas medicinais e $75,9 \%$ fitoterápicos. Além disso, o uso concomitante foi relatado por $72,3 \%$ das idosas entrevistadas. Entre os homens, $27,5 \%$ utilizam plantas medicinais, $24,1 \%$ fitoterápicos e $22,7 \%$ afirmam usar ambos os tratamentos. Estudos revelam que essa predominância feminina no uso de plantas medicinais se deve ao fato de as mulheres serem consideradas as principais responsáveis pelos cuidados caseiros, e muitas vezes estarem envolvidas diretamente no cuidado e tratamento dos membros da família, além de terem maiores conhecimentos sobre o assunto devido à prática de trocar mudas e receitas de remédios caseiros ${ }^{29,30}$.

O uso concomitante de medicamentos alopáticos e plantas medicinais pelos idosos entrevistados em Ituiutaba/MG é relativamente baixo se comparado ao estudo realizado por Machado et al. ${ }^{28}$ em Uberlândia/ MG, em que $86,2 \%$ e $81,3 \%$ dos idosos utilizam simultaneamente plantas medicinais e fitoterápicos com medicamentos convencionais, respectivamente. Os espécimes mais mencionados pelos idosos que afirmaram usar plantas medicinais foram erva-cidreira, hortelā, erva-de-santa-maria, boldo, canela e alecrim, além de chás preparados com plantas das quais os entrevistados desconhecem o nome. As espécies não foram coletadas para identificação, uma vez que $o$ interesse era apenas no conhecimento popular sobre seu uso. As principais indicaçóes para tratamento com plantas medicinais foram como analgésico, antitérmico, relaxante muscular, anti-inflamatório e antigripal.
Por outro lado, o tratamento com medicamentos fitoterápicos foi citado, principalmente, para dores articulares e musculares, sintomas de gripe e infecçóes no trato respiratório, labirintite, diabetes e ansiedade.

$\mathrm{O}$ uso de algumas dessas plantas também foi encontrado em estudos de Brasileiro et al. ${ }^{31}$, Araújo et al. $^{30}$ e Ângelo e Ribeiro ${ }^{32}$. Os trabalhos de Brasileiro et al. ${ }^{31}$ e Pires et al. ${ }^{29}$ relatam o uso das plantas medicinais para o tratamento de doenças nos sistemas respiratório, cardiovascular e digestivo, além de lesôes, infecçôes e estresse. De acordo com Machado et al. ${ }^{33}$, plantas medicinais podem desencadear reaçóes adversas com a interação de medicamentos, sendo necessária a elaboração de estratégias interventivas a fim de informar os idosos sobre os riscos do uso combinado de plantas medicinais e fármacos ${ }^{33}$. É importante salientar que o uso de duas ou mais plantas medicinais é caracterizado como poliherbácea e, apesar de $70 \%$ da populaçáo idosa relatar ter pelo menos uma condição crônica e de o uso de automedicaçáo e a associação de terapias serem uma realidade, são imprescindíveis a capacitação do profissional de saúde e o desenvolvimento de estratégias de divulgação que apoiem as experiências de educaçáo e saber popular, bem como a promoçáo da prescriçáo segura e o fornecimento de fitoterápicos na rede pública de saúde para diminuição do custo no tratamento para os idosos ${ }^{34}$.

O número mínimo de medicamentos alopáticos que os idosos entrevistados utilizavam foi um e o máximo, cinco, sendo que a maioria $(51,7 \%)$ fazia uso de dois a quatro medicamentos. É considerado polifarmácia o uso de cinco ou mais medicamentos alopáticos, e somente $26,1 \%$ dos idosos declararam realizar essa prática. No entanto, a pré-polifarmácia, definida pelo uso de três ou mais medicamentos alopáticos, foi registrada para $51,7 \%$ dos entrevistados (Tabela 2). Tal resultado supera o encontrado por Silva et al. ${ }^{35}$, em que a polifarmácia ocorreu em mais de um terço dos entrevistados. Esse mesmo estudo relata que dados nacionais revelam prevalência de polifarmácia entre $10,9 \%$ a $38 \%$ dos idosos, com média de utilizaçáo entre dois e cinco medicamentos. Vale ressaltar que, após análise estatística com os dados dos idosos entrevistados em Ituiutaba/MG, constatou-se que o uso de medicamentos alopáticos está associado ao número de doenças crônicas diagnosticadas pelos médicos $(p \leq 0.0001)$.

Em relação ao número de doenças autorreferidas, obteve-se média de 1,8 por idoso entrevistado (mín: 1; máx: $5 ; \pm 1,06$ ). Além disso, cerca de $90 \%$ da amostra foi diagnosticada pelos médicos, sendo mais prevalentes entre as mulheres $(62,4 \%)$, especialmente com idade de 60 a 69 anos (43,6\%) (Gráfico 1). Quanto aos tipos de 
doenças autorreferidas, houve maior ocorrência do sistema circulatório $(75,8 \%)$, destacando-se a hipertensão arterial $(72,5 \%)$ em todas as UBSF (Tabela 3). Para o sistema endócrino (39,9\%), destacou-se o diabetes mellitus, sendo que $21,8 \%$ dos idosos náo sabiam qual era o tipo. Ainda, 41,6\% dos idosos relataram possuir algum problema no sistema osteomuscular, exceto na UBSF 8 (Tabela 3).

A alta frequência de idosos com disfunção no sistema endócrino e circulatório apresentou significância em outros trabalhos ${ }^{36-39}$. De acordo com Santos et al. ${ }^{19}$, de 207 idosos usuários da Estratégia Saúde da Família do município de Santa Juliana/MG evidenciou-se que $71 \%$ foram diagnosticados com hipertensão arterial, enquanto $18,4 \%$ apresentavam diabetes e $31,4 \%$, doenças no sistema osteomuscular. White e León ${ }^{40}$ discutem a necessidade de estimular a população com açôes de educação em saúde, permitindo, assim, a autonomia nas escolhas, nas decisóes e nos comportamentos em relação ao tratamento.

Gráfico 1: Grupos de doenças, classificadas conforme o CID, autorreferidas por idosos atendidos nas Unidades Básicas de Saúde da Família (UBSF), segundo faixa etária e sexo, Ituiutaba/MG, 2015

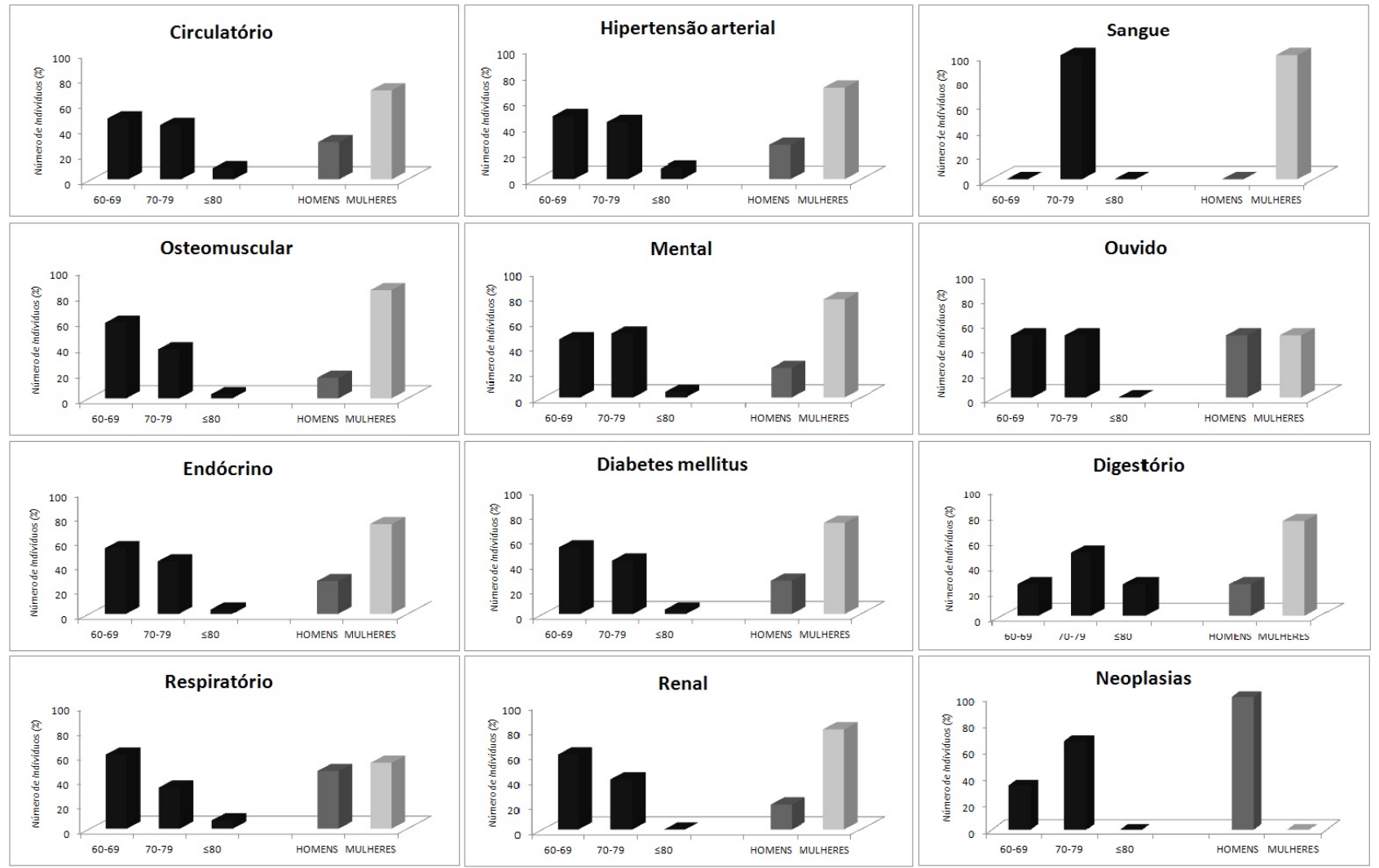

Fonte: Elaborado pelos autores (2015).

De acordo com Saraiva et $\mathrm{al}^{39}$, a hipertensão arterial e o diabetes mellitus são doenças multifatoriais, tendo em vista que as estratégias de combate a elas devem ser variadas, com base em suas diversas configuraçóes. Uma das principais dificuldades encontradas pelo sistema de saúde é a detecção precoce para minimizar a incidência de futuras complicaçóes, tanto na população de alto risco quanto na população em geral ${ }^{39}$. Contudo, é importante ressaltar que, neste grupo populacional, Melo et al. ${ }^{2}$ descobriram alto índice de diabéticos e hipertensos que não seguem as orientaçóes ou não consideram sua alimentação saudável, indicando baixa adesão às recomendaçóes nutricionais. Dessa maneira, açóes estratégicas de profissionais de saúde que envolvam toda a comunidade são relevantes para mudanças de hábitos, promovendo a alimentação saudável e contribuindo para a prevenção e o tratamento de doenças crônicas².

As demais doenças autorreferidas pelos idosos foram classificadas segundo a Classificaçáo Internacional de Doenças (CID), destacando-se os problemas mentais 
Tabela 3: Doenças crônicas autorreferidas por idosos atendidos nas Unidades Básicas de Saúde da Família (UBSF), Ituiutaba/MG, 2015

\begin{tabular}{|c|c|c|c|c|c|c|c|c|c|c|c|c|c|c|c|c|c|c|c|c|c|c|c|c|}
\hline \multirow{2}{*}{$\begin{array}{l}\text { Doenças } \\
\text { Crônicas }\end{array}$} & \multicolumn{2}{|c|}{$\begin{array}{c}\text { Total } \\
(n=149)\end{array}$} & \multicolumn{2}{|c|}{$\begin{array}{l}\text { UBSF } 1 \\
(n=14)\end{array}$} & \multicolumn{2}{|c|}{$\begin{array}{l}\text { UBSF } 2 \\
(n=13)\end{array}$} & \multicolumn{2}{|c|}{$\begin{array}{l}\text { UBSF } 3 \\
(n=13)\end{array}$} & \multicolumn{2}{|c|}{$\begin{array}{l}\text { UBSF } 4 \\
(n=13)\end{array}$} & \multicolumn{2}{|c|}{$\begin{array}{l}\text { UBSF } 5 \\
(\mathbf{n}=15)\end{array}$} & \multicolumn{2}{|c|}{$\begin{array}{l}\text { UBSF } 6 \\
(n=13)\end{array}$} & \multicolumn{2}{|c|}{$\begin{array}{l}\text { UBSF } 7 \\
(\mathbf{n}=14)\end{array}$} & \multicolumn{2}{|c|}{$\begin{array}{l}\text { UBSF } 8 \\
(n=14)\end{array}$} & \multicolumn{2}{|c|}{$\begin{array}{l}\text { UBSF } 9 \\
(n=13)\end{array}$} & \multicolumn{2}{|c|}{$\begin{array}{l}\text { UBSF } 10 \\
(n=14)\end{array}$} & \multicolumn{2}{|c|}{$\begin{array}{c}\text { UBSF 11 } \\
(n=13)\end{array}$} \\
\hline & $n$ & $\%$ & $n$ & $\%$ & $n$ & $\%$ & $n$ & $\%$ & $n$ & $\%$ & $n$ & $n$ & $n$ & $\%$ & $n$ & $\%$ & $n$ & $\%$ & $n$ & $\%$ & $n$ & $\%$ & $n$ & $\%$ \\
\hline & & & 10 & & 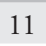 & & 12 & & 11 & & 14 & 10 & 10 & & 9 & & 12 & & 10 & 76,9 & & & 10 & 76,9 \\
\hline & & & 0 & & & & 2 & & 0 & & 11 & & 0 & & & 4,3 & 2 & & 7 & 3,8 & & & & 61,5 \\
\hline & - & & 0 & 0 & 0 & 0 & 0 & 0 & & 0 & & & & 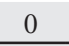 & & & & & & & & & & 0 \\
\hline & & & 5 & & 6 & & 5 & & 5 & & & 6 & 6 & & 9 & & & & 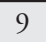 & & & & & 6,2 \\
\hline & & & 4 & 26 & & & & 0 & & & & & $J$ & & & 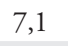 & & & & 7 & & & & 3,1 \\
\hline & 4 & & U & 0 & 0 & 0 & 0 & & & & & & & & & & & & & & & & & 0 \\
\hline & & & 6 & & 7 & & 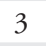 & & & & & & & & & & & & & & & & & 54 \\
\hline ellitus & 2 & & 0 & 0 & 1 & & 0 & 0 & 0 & 0 & 0 & 0 & 0 & 0 & 0 & 0 & 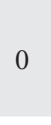 & 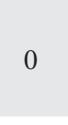 & 1 & 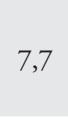 & 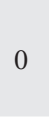 & 0 & & 0 \\
\hline & & & 3 & & 0 & 0 & - & & & & & 0 & ( & & 2 & & & & & 0 & & & & 0 \\
\hline & 9 & & 0 & 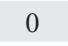 & 1 & & 2 & & & & & 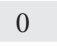 & & & 2 & & 1 & & 2 & & & & & 列 \\
\hline$\therefore$ & 31 & $300^{\circ}$ & 3 & & 5 & & 3 & & & 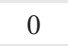 & & 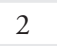 & 2 & & 2 & 14,3 & 4 & & 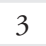 & 23,1 & ${ }^{-}$ & & L & 15,4 \\
\hline & 4 & & 0 & 0 & 1 & & 1 & & & & & 0 & - & & & & & & 0 & 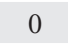 & & & & 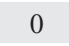 \\
\hline & 15 & & 2 & & 2 & & 3 & & 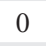 & & & ( & 3 & 15,4 & & & & & 2 & 15,4 & 1 & & 3 & 23,1 \\
\hline & 5 & 3.4 & 0 & 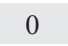 & 1 & & 0 & . & 0 & & & 0 & 0 & 0 & & & & & 2 & 15,4 & & 0 & & 0 \\
\hline veopiasias & 3 & 2,0 & 0 & 0 & 1 & & 0 & 0 & 0 & & & 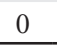 & 0 & $7, /$ & 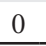 & 0 & 0 & & 0 & a & & & - & 0 \\
\hline
\end{tabular}

Fonte: Elaborado pelos autores (2015).

$(14,8 \%)$, com maior frequência nas UBSF $1(28,6 \%), 6$ $(30,8 \%)$ e $11(23,1 \%)$; no sistema respiratório $(10,1 \%)$; renais $(3,4 \%)$, encontrados nas UBSF 2,5 e 9; problemas no ouvido (2,7\%); no sistema digestório $(2,7 \%)$, relatados nas UBSF 2, 3, 5 e 6; sanguíneos $(0,7 \%)$, somente na UBSF 10, na faixa etária de 70 a 79 anos; e neoplasias (2,0\%), com representatividade nas UBSF 2, 5 e 6 (Tabela 3). Esses dados corroboram os resultados encontrados na Estratégia de Saúde da Família da regiāo Sul do Brasil ${ }^{40}$, em que as doenças no aparelho respiratório e os transtornos mentais e comportamentais foram maioria, enquanto neoplasias e problemas sanguíneos foram minoria.

Segundo o Censo Demográfico do IBGE $^{40}$ de 2014 em Ituiutaba/MG, no item "Morbidades Hospitalares", de um total de 421 óbitos, a maioria dos registros foi para as doenças dos aparelhos respiratório $(29,2 \%)$ e circulatório $(18,8 \%)$. Em menor frequência houve registro de óbitos por neoplasias $(9,3 \%)$, doenças endócrinas, nutricionais e metabólicas $(4,8 \%)$; problemas sanguíneos, nos órgãos hematológicos e transtornos imunitários (1,9\%); transtornos mentais e comportamentais $(0,5 \%)$; doenças osteomusculares e no tecido conjuntivo $(0,25 \%)$. Apesar de as prevalências encontradas náo serem as mesmas em relação às doenças investigadas, é importante considerar que, no registro do IBGE, não há distinção de idade. Além disso, sabemos que muitas dessas doenças podem não levar os idosos ao óbito, mas são consideradas comorbidades de outras enfermidades adquiridas ao longo da vida. Vale chamar a atenção para o fato de que nem todas as internações e mortes são registradas, havendo uma negligência na área da saúde.

Ao avaliar a percepção da saúde pelos idosos entrevistados em Ituiutaba/MG, percebe-se que a maioria a considera razoável (46,3\%) (Tabela 4). No Gráfico 2, é possível observar que, em relação à idade, a percepção da saúde como razoável prevaleceu para a faixa etária de 60 a 69 anos (56,2\%), enquanto a razoável e boa ficou para os idosos de 70 a 79 anos (36,5\%), e boa para idosos com oitenta anos ou mais $(46,2 \%)$. Ao comparar a percepção da saúde entre os gêneros, constata-se que a maioria dos homens e mulheres percebe sua saúde como razoável (44,0\% e 47,5\%, respectivamente). Entre os idosos atendidos nas UBSF 1 e 3 prevaleceu a percepção da saúde como boa ( 42,9 e $53,8 \%$, respectivamente). Interessantemente, houve maior predominância da percepçáo da saúde como ótima somente na UBSF 6 $(38,5 \%)$ (Tabela 4). 
Tabela 4: Percepção da saúde dos idosos atendidos nas Unidades Básicas de Saúde da Família (UBSF), Ituiutaba/MG, 2015

\begin{tabular}{|c|c|c|c|c|c|c|c|c|c|c|c|c|c|c|c|c|c|c|c|}
\hline \multirow{2}{*}{$\begin{array}{l}\text { Percepçáo } \\
\text { da saúde }\end{array}$} & \multicolumn{2}{|c|}{$\begin{array}{c}\text { Total } \\
(n=149)\end{array}$} & $\begin{array}{l}\text { UBSF } 1 \\
(n=14)\end{array}$ & $\begin{array}{l}\text { UBSF } 2 \\
(n=13)\end{array}$ & \multicolumn{2}{|c|}{$\begin{array}{l}\text { UBSF } 3 \\
(n=13)\end{array}$} & $\begin{array}{l}\text { UBSF } 4 \\
(n=13)\end{array}$ & $\begin{array}{l}\text { UBSF } 5 \\
(\mathrm{n}=15)\end{array}$ & $\begin{array}{l}\text { UBSF } 6 \\
(n=13)\end{array}$ & \multicolumn{2}{|c|}{$\begin{array}{l}\text { UBSF } 7 \\
(n=14)\end{array}$} & \multicolumn{2}{|c|}{$\begin{array}{l}\text { UBSF } 8 \\
(n=14)\end{array}$} & \multicolumn{2}{|c|}{$\begin{array}{l}\text { UBSF } 9 \\
(n=13)\end{array}$} & \multicolumn{2}{|c|}{$\begin{array}{l}\text { UBSF } 10 \\
(n=14)\end{array}$} & \multicolumn{2}{|c|}{$\begin{array}{c}\text { UBSF } 11 \\
(n=13)\end{array}$} \\
\hline & $n$ & $\%$ & $\%$ & $\%$ & 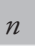 & $\%$ & $n$ & $\%$ & $\%$ & - & $\%$ & $n$ & $\%$ & . & $\%$ & $n$ & $\%$ & $n$ & $\%$ \\
\hline & 21 & 4,1 & 321,4 & 215,4 & 3 & 3 , & 1 & 0 & 0 & 0 & 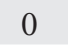 & 1 & 1 & 1 & 7 & 2 & 3 & 4 & 8 \\
\hline & 69 & 16,3 & $4 \quad 28,6$ & 646,2 & 2 & 15,4 & 969,2 & 960,0 & 3,8 & f & 50,0 & 10 & 71,4 & 7 & 53,8 & 5 & 5,7 & 6 & 46,2 \\
\hline 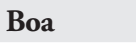 & 43 & 28,9 & $6 \quad 42,9$ & $4 \quad 30,8$ & 7 & 53,8 & 1 & $4 \quad 26,7$ & $4 \quad 30,8$ & 6 & 42,9 & 1 & 7,1 & 3 & 1 & 5 & 7 & 1 & 7,7 \\
\hline Ótima & 16 & 10,7 & 17,1 & 7,7 & 1 & 7,1 & 215,4 & 213,3 & 538,5 & 1 & 7,1 & 2 & 14,3 & 2 & 15,4 & 2 & 14,3 & 2 & 15,4 \\
\hline
\end{tabular}

Fonte: Elaborado pelos autores (2015).

Gráfico 2: Percepção da saúde dos idosos atendidos nas Unidades Básicas de Saúde da Família (UBSF), segundo faixa etária e sexo, Ituiutaba/MG, 2015

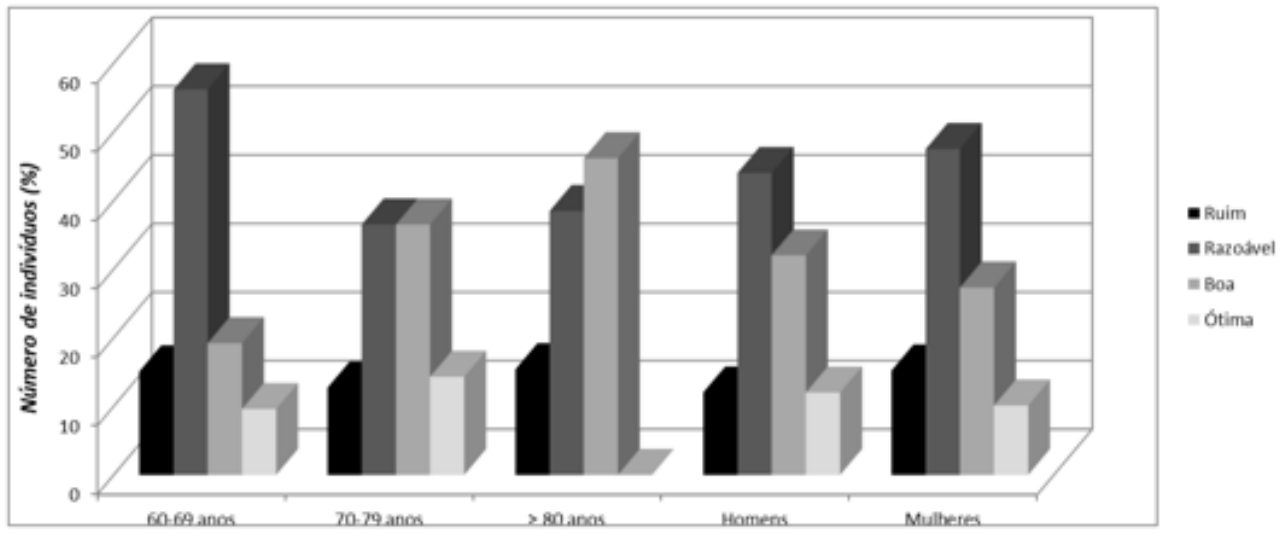

Fonte: Elaborado pelos autores (2015).

Segundo os resultados do estudo de Fernandes et al. ${ }^{36}$, realizado no Programa Saúde da Família de João Pessoa/PB, 54\%, dos idosos percebiam sua saúde como regular, $27,7 \%$ a descreviam como boa/muito boa e $18,3 \%$ como ruim/muito ruim. Esses resultados também corroboram os obtidos por Silva et al. ${ }^{35} \mathrm{em}$ Quixadá/CE, onde $75,5 \%$ dos idosos definiam sua saúde como regular. De acordo com Teston, Caldas e $\operatorname{Marcon}^{41}$, a autoavaliação de saúde é considerada um indicador importante da condição de saúde de indivíduos e populaçooses, isto é, idosos que avaliam a própria saúde como ruim apresentam risco elevado de mortalidade e aumento do índice de internaçóes em comparaçáo com aqueles que classificam sua saúde como excelente. Nesse sentido, devemos estimular o desenvolvimento de autopercepção positiva de vida e saúde nos idosos, uma vez que essa atitude pode ser um fator de proteção.

É importante salientar que os dados deste estudo foram obtidos por meio de questionários autorresponsivos, o que pode constituir uma limitação, uma vez que foram contabilizados apenas quando os idosos possuíam alguma DCNT e utilizavam medicamentos alopáticos, plantas medicinais e fitoterápicos por autodeclaração, sem testes clínicos para comprovar o diagnóstico dessas doenças e sem acesso às cadernetas de saúde desses pacientes.

\section{Conclusão}

Diante dos dados coletados, é possível caracterizar a situação da população idosa residente em Ituiutaba/ MG e atendida nas Unidades Básicas de Saúde da Família (UBSF) como de indivíduos fragilizados, pois sáo idosos, polimedicados, com baixo grau de instrução e, pelo menos, uma DCNT, com destaque para as doenças dos sistemas circulatório, endócrino e osteomuscular. Houve maior prevalência do uso de fitoterápicos e plantas medicinais entre as mulheres. Ademais, quanto maior a utilização de polifarmácia concomitantemente com poliherbácia, pior é a percepção da saúde do idoso investigado. Esses dados revelam a importância de estratégias e ações dos profissionais de 
saúde que envolvam toda a comunidade, incluindo os idosos e suas famílias, com o intuito de reverter esse quadro. Além do mais, espera-se que este estudo sirva de apoio para o Ministério da Saúde, a Secretaria Regional de Saúde e a Secretaria Municipal de Saúde no desenvolvimento de açóes direcionadas à população idosa e que tais orgãos públicos vislumbrem o envelhecimento saudável e ativo.

\section{Agradecimentos}

Aos estudantes Camila Aparecida Marques Silva, Laura Maria Araújo Vêncio, Matheus Moura Martins e Mayalla de Freitas Faria por auxiliarem na coleta de dados e visita às Unidades Básicas de Saúde da Família. À Prefeitura Municipal de Ituiutaba/Secretaria Municipal de Saúde. Ao apoio financeiro concedido pelo Programa de Extensão, Integração UFU/Comunidade, via PróReitoria de Extensão, Cultura e Assuntos Estudantis da UFU, por meio de aprovação do projeto "Atenção preventiva e educativa em saúde do idoso: o saber e o fazer compartilhados em Ituiutaba/MG” (SIEX 12440).

\section{Referências}

1. Campos LM, Boscatto EC, Mineiro L. Perfil do estilo de vida dos idosos da Universidade Aberta da Maior Idade - UAMI da cidade de Caçador-SC. Revista Brasileira de Prescrição e Fisiologia do Exercício. 2015;9(53):315-20.

2. Melo JV, Santos ALM, Rezende AAA, Calábria LK. Hábitos alimentares dos idosos atendidos nas Unidades Básicas de Saúde da Família (UBSF) em ItuiutabaMG. Rev Med Saúde Brasília. 2017; 6(2): 154-66.

3. Zani VT, Broilo MC, Lando VR, Schneider RH. Intervenção educativa e alimentar com suplemento de aveia em mulheres idosas. Caderno Pedagógico. 2015;12(1):184-95.

4. Dutra DD, Duarte MCS, Albuquerque KF, Lima AS, Santos JS, Souto HC. Doenças cardiovasculares e fatores associados em adultos e idosos cadastrados em uma unidade básica de saúde. J Res: Fundam Care. 2016;8(2):4501-9.

5. Franco LG, Kindermann AL, Tramujas L, Kock KS. Fatores associados à mortalidade em idosos hospitalizados por fraturas de fêmur. Rev Bras Ortop. 2016;51(5):509-14.

6. Leite MT, Dal-Pai S, Quintana JM, Costa MC. Doenças crônicas não transmissíveis em idosos: saberes e açôes de agentes comunitários de saúde. J Res: Fundam Care. 2015;7(2):2263-76.

7. Ramos LR, Tavares NUL, Bertoldi AD, Farias MR, Oliveira MA, Luiza VL, et al. Polifarmácia e polimorbidade em idosos no Brasil: um desafio em saúde pública. Rev Saúde Pública. 2016;50(supl. 2):1-13.
8. Instituto Brasileiro de Geografia e Estatística. Coordenação de População e Indicadores Sociais. Síntese de indicadores sociais: uma análise das condições de vida da população brasileira. Rio de Janeiro: IBGE; 2012 (Estudos e Pesquisas; no. 29).

9. Instituto Brasileiro de Geografia e Estatística. Coordenação de Populaçáo e Indicadores Sociais. Síntese de indicadores sociais: uma análise das condiçóes de vida da população brasileira. Rio de Janeiro: IBGE; 2013 (Estudos e Pesquisas; no. 32).

10. Anderson GM, Beers MH, Kerluke K. Auditing prescription practice using explicit criteria and computerized drug benefit claims data. J Eval Clin Pract. 1997;3(4):283-94.

11. Loyola Filho AI, Matos DL, Giatti L, Alfradique ME, Peixoto SV, Lima-Costa MF. Causas de internaçóes hospitalares entre idosos brasileiros no âmbito do Sistema Único de Saúde. Epidemiol Serv Saúde. 2004;13(4):229-38.

12. Mendes TAB. Prevalência de doenças crônicas e a utilizaçáo dos serviços de saúde por idosos residentes no município de São Paulo [tese]. São Paulo: Universidade de São Paulo; 2010.

13. Magalhães BS, Ibiapina DF, Carvalho DR. Avaliação nutricional e prevalência de diabetes e hipertensão em idosos. Rev Interd. 2014;7(4):131-38.

14. Marques LP, Confortin SC. Doenças do aparelho circulatório: principal causa de internaçóes de idosos no Brasil entre 2003 e 2012. Rev Bras Ciênc Saúde. 2015;19(2):83-90.

15. Brasil. Ministério da Saúde. Secretaria de Vigilância em Saúde. Departamento de Análise de Situação de Saúde. Plano de açóes estratégicas para o enfrentamento das doenças crônicas não transmissíveis (DCNT) no Brasil 20112022. Brasília: Ministério da Saúde; 2011.

16. Deon RG, Rosa RD, Zanardo VPS, Closs VE, Schwanke CHA. Consumo de alimentos dos grupos que compóem a pirâmide alimentar americana por idosos brasileiros: uma revisão. Ciência \& Saúde. 2015;8(1):26-34.

17. Gadenz SD, Benvegnu LA. Hábitos alimentares na prevenção de doenças cardiovasculares e fatores associados em idosos hipertensos. Ciên Saúde Coletiva. 2013;18(12):3523-33.

18. Lima-Costa MF, Barretos SM. Tipos de estudos epidemiológicos: conceitos básicos e aplicaçóes na área do envelhecimento. Epidemiol Serv Saúde. 2003;12(4):189-201.

19. Santos AS, Meneguci J, Silveira RE, Marquez FA, Ferreira VA, Viana DA. Perfil de saúde e qualidade de vida de idosos de Santa Juliana-MG. Enferm Foco. 2014;5(3/4):61-4.

20. Kuznier TP, Souza CC, Chianca TCM, Ercole FF, Alves M. Fatores de risco para quedas descritos na taxonomia da NANDA-I para uma população de idosos. Rev Enferm Cent O Min. 2015;5(3):1855-70.

21. Siqueira FV, Facchini LA, Piccini RX, Tomasi E, Thumé E, Silveira DS, et al. Prevalência de quedas em idosos e fatores associados. Rev Saúde Pública. 2007;41(5):749-56.

22. Maciel A. Quedas em idosos: um problema de saúde pública desconhecido pela comunidade e negligenciado por muitos profissionais da saúde e por autoridades sanitárias brasileiras. Rev Med Minas Gerais. 2010;20(4):554-7. 
23. Fabrício SCC, Rodrigues RAP, Junior MLC. Causas e consequências de quedas de idosos atendidos em hospital público. Rev Saúde Pública. 2004;38(1):93-9.

24. Soares DS, Mello LM, Silva AS, Nunes AA. Análise dos fatores associados a quedas com fratura de fêmur em idosos: um estudo caso-controle. Rev Bras Geriatr Gerontol. 2015;18(2):239-48.

25. Alves ECS, Souza LPS, Alves WS, Oliveira MKS, Yoshitome AY, Gamba MA. Condições de saúde e funcionalidade de idosos com diabetes mellitus tipo 2 na Atenção Primária à Saúde. Enfermería Global. 2014;13(34):19-31.

26. Silva GOB, Gondim APS, Monteiro MP, Frota MA, Meneses ALL. Uso de medicamentos contínuos e fatores associados em idosos de Quixadá, Ceará. Rev Bras Epidemiol. 2012;15(2):386-95.

27. Sardinha AHL, Silva CG, Sena LB, Mesquita LLS, Rodrigues JB, Silva KNR. Adesão dos idosos com doenças crônicas ao tratamento medicamentoso. Rev Pesq Saúde. 2015;16(3):154-58.

28. Machado HL, Moura VL, Gouveia NM, Costa GA, Espindola FS, Botelho FV. Pesquisa e atividades de extensão em fitoterapia desenvolvidas pela Rede FitoCerrado: uso racional de plantas medicinais e fitoterápicos por idosos em Uberlândia-MG. Rev Bras Plantas Med. 2014;16(3):527-33.

29. Pires IFB, Souza AA, Feitosa MHA, Costa SM. Plantas medicinais como opção terapêutica em comunidade de Montes Claros, Minas Gerais, Brasil. Rev Bras Plantas Med. 2014;16(2):426-33.

30. Araújo CRF, Silva AB, Tavares EC, Costa EP, Mariz SR. Perfil e prevalência de uso de plantas medicinais em uma unidade básica de saúde da família em Campina Grande, Paraíba, Brasil. Rev Ciênc Farm Básica Apl. 2014;35(2):233-38.

31. Brasileiro BG, Pizziolo VR, Matos DS, Germano AM, Jamal CM. Plantas medicinais utilizadas pela população atendida no "Programa de Saúde da Família”, Governador Valadares, MG, Brasil. Rev Bras Ciênc Farm. 2008;44(4):629-36.

32. Ângelo $T$, Ribeiro CC. Utilização de plantas medicinais e medicamentos fitoterápicos por idosos. C\&D-Revista
Eletrônica da Fainor. 2014;7(1):18-31.

33. Machado RS, Pijuán PL, Brum VS, Moreira APG, Oliveira LFS, Farias F. Avaliação sobre o conhecimento do uso de plantas medicinais em dois grupos de idosos. Anais do 7 . Salão Internacional de Ensino, Pesquisa e Extensão, Bagé, Rio Grande do Sul: Unipampa, 2015.

34. Calabria LK, De Rezende CHA. Plantas medicinais: desafios da prática do saber popular no envelhecimento. In: Faria L, Calábria LK, Alves WA. Envelhecimento: um olhar interdisciplinar. São Paulo: Hucitec; 2016. p. 322-53.

35. Silva AL, Ribeiro AQ, Klein $\mathrm{CH}$, Acurcio FA. Utilização de medicamentos por idosos brasileiros, de acordo com a faixa etária: um inquérito postal. Cad Saúde Pública. 2012; 28(6):1033-45.

36. Fernandes MGM, Souto MC, Costa SFG, Fernandes BM. Qualificadores sócio-demográficos, condiçóes de saúde e utilizaçáo de serviços por idosos atendidos na atençáo primária. Rev Bras Ciênc Saúde. 2009;13(2):13-20.

37. Brasil. Ministério do Planejamento, Orçamento e Gestão. Instituto Brasileiro de Geografia e Estatística. Diretoria de Pesquisas. Coordenação de Trabalho e Rendimento. Pesquisa nacional de saúde: percepção do estado de saúde, estilos de vida e doenças crônicas - Brasil, grandes regióes e unidades da federação. Rio de Janeiro: IBGE; 2013.

38. Wendt CJK, Aires M, Paz AA, Fengler FL, Paskulin LMG. Famílias de idosos na Estratégia de Saúde no sul do Brasil. Rev Bras Enferm. 2015;68(3):406-13.

39. Saraiva LGF, Dornelas PG, Cau SBA, Calábria LK. Perfil epidemiológico de pacientes atendidos em uma rede ambulatorial do Hiperdia Minas em Governador Valadares-MG. Rev. Aten. Saúde. 2016;14(48):40-7.

40. White HJ, Marín-León L. Orientaçóes nutricionais em serviços de saúde: a percepçáo de idosos portadores de hipertensão e diabetes. Demetra. 2014;9(4):867-88.

41. Teston EF, Caldas CP, Marcon SS. Condomínio para idosos: condiçôes de vida e saúde de residentes nesta nova modalidade habitacional. Rev Bras Geriatr Gerontol. 2015;18(3):487-97.

\section{Como citar este artigo:}

Melo JV, Santos ALM, Faria LR, Alves WA, Rezende AAA, Calábria LK. Perfil de saúde dos idosos atendidos nas Unidades Básicas de Saúde da Família (UBSF) em Ituiutaba, Minas Gerais. Rev. Aten. Saúde. 2017;15(53):66-75. 